\title{
En torno a la nueva visión de Husserl
}

Javier San Martín

UNED, España

\section{Resumen}

El presente texto es un extracto de la primera lección del curso impartido en Guanajuato en la primavera del año en curso. $\mathrm{Su}$ objetivo es determinar los contenidos que configuraron la interpretación convencional de Husserl, para destacar lo que sería el objetivo del curso: la noción del "nuevo" Husserl. El ensayo consta de cinco apartados. En el primero se presenta el contenido global de la obra pública de Husserl. El segundo se dedica a explicar la noción del "nuevo" Husserl. El apartado tres procura exponer los rasgos del Husserl convencional. El cuatro está dedicado a ver la cadena de autores que nos han transmitido al "nuevo" Husserl. Para terminar, en el apartado quinto se hace referencia a algunos textos de Gaos sintomáticos de la comprensión convencional de Husserl.

Palabras clave: fenomenología husserliana, crítica de Heidegger, reflexión, crítica de Gaos, historia de la fenomenología. 


\section{Abstract}

The paper is an excerpt from the first lesson of the course in Guanajuato in the spring of this year. Its aim is to determine the content of the standard interpretation of Husserl, to highlight the concept of "new" Husserl, what will be understood later and that was the aim of the lectures. The test consists of five sections. The first presents the overall content of the public work of Husserl. The second section is devoted to explaining the concept of "new" Husserl. Paragraph 3 seeks to expose the features of conventional Husserl. Paragraph 4 is devoted to see the chain of authors who have given us the "new" Husserl, and, finally, in Paragraph 5, it is exposed a reference to certain texts of Gaos that are very symptomatic of the conventional understanding of Husserl.

Keywords: Husserl's Phenomenology, Heidegger's criticisme; Reflexion, Gaos' criticisme; history of phenomenology.

T as siguientes páginas tienen como objetivo ofrecer la proble_mática general de la recepción de Husserl, en la medida en que su obra, que globalmente está detrás de algunos de los desarrollos filosóficos más importantes del siglo XX, ha sido por lo general recibida de modo desenfocado. El hecho de iniciar con la fenomenología una nueva orientación de la filosofía, oculta el nuevo significado de las palabras en que esa nueva filosofía se anuncia. Además, Husserl publicó sólo una mínima parte de lo que escribió, por lo que era muy difícil entender realmente el verdadero sentido de su filosofía. Así, su recepción trascurrió por dos caminos: uno, el convencional, profundamente desenfocado, y otro, que proviene de sus discípulos de Friburgo, pero que sólo a principios del siglo XXI ha terminado por aparecer a la luz pública con el nombre de "el nuevo Husserl". 
1. La obra pública de Husserl y la crítica de Heidegger

Al estudiar manuscritos de Husserl, cuando eso fue posible en los años cincuenta y luego ya en los siguientes años sesenta y setenta, se tenía el convencimiento de que se estaba leyendo algo distinto de lo que se podía encontrar en los manuales o incluso en muchos de los textos en los que hablaban de fenomenología, es decir, en los textos de algunos investigadores que parecían ofrecer garantías de conocimiento de la filosofía de Husserl. ${ }^{1}$ Así, en el más inmediato y primer contacto con los textos aún no publicados de Husserl, nos encontrábamos un Husserl que parecía distinto o que no se compaginaba con lo que leíamos en otros sitios. Incluso podríamos tener la sensación de que el Husserl de los manuscritos era distinto del Husserl de los libros publicados o, al menos, que esos manuscritos daban a lo publicado un sentido distinto del que podía tener sin tomar en consideración esos manuscritos. Cuando, por otro lado, ya más metido en la obra de Ortega y Gasset, leí las críticas de éste a Husserl, o las que le hacían tanto Antonio Rodríguez Huéscar, ya en $1982,{ }^{2}$ como Julián Marías, di por hecho que lo criticado se refería al Husserl de los libros publicados, pero que esas críticas no llegaban a un Husserl matizado por los escritos inéditos. Un buen ejemplo es el tema de la intersubjetividad, para el que la crítica tomaba como referencia la "V Meditación cartesiana", pero que en los manuscritos de Husserl tiene referencias mucho más amplias ya desde 1910, en las lecciones sobre Los problemas fundamentales de la fenomenología.

${ }^{1}$ Es el caso de la presentación de Husserl que hace Zubiri en Cinco lecciones de filosofia. Zubiri había hecho la tesis doctoral sobre el psicologismo en Husserl, contando naturalmente con las obras publicadas hasta ese momento.

${ }^{2}$ La innovación metafísica de Ortega: crítica y superación del idealismo (Rodríguez Huéscar, 1982). Hay edición de 2002, preparada por Jorge García Gómez en Biblioteca Nueva. 
Tenemos, por tanto, un Husserl convencional, basado en las obras publicadas a lo largo de su vida, que eran muy pocas. Es conveniente hacerse una idea muy precisa de esta obra publicada. La vida profesional de Husserl transcurre en tres universidades: la de Halle, la de Gotinga y la de Friburgo. ${ }^{3}$ Cada una de estas épocas está caracterizada por alguna de las importantes publicaciones de Husserl. En la época de Halle, que termina con la publicación de las Investigaciones lógicas, vio la luz, además del escrito de $\mathrm{Ha}$ bilitación (1887), la Filosofía de la aritmética (1891). En el periodo de Gotinga, luego del largo artículo publicado en la revista Logos, "La filosofía como ciencia estricta", se publica el libro fundamental Ideas para una fenomenología pura y una filosofía fenomenológica (1913). En el tercer periodo, el de Friburgo, ${ }^{4}$ tenemos, al final del mismo, el libro básico La crisis de las ciencias europeas y la fenomenología trascendental, aunque publicado sólo de modo parcial. Como veremos, la etapa de Friburgo, que abarca los últimos veintidós años de la vida de Husserl, puede ser dividida en dos partes: una desde que viene llamado de Gotinga hasta la jubilación con un año de adelanto en 1928, y, la otra, la etapa de jubilado. La primera, que como se verá está dedicada en gran medida a la filosofía práctica, termina con la publicación de La lógica formal y trascendental (1929). El año anterior se habían publicado las primeras Lecciones sobre la conciencia interna del tiempo y, un poco después, aunque sólo en francés, Meditaciones cartesianas (1931).

Y con estas pocas líneas he contado las publicaciones más importantes y conocidas de Husserl hechas en su vida. Ahora, mi-

\footnotetext{
${ }^{3}$ Esa vida profesional fue el tema de la sesión segunda.

${ }^{4}$ Las sesiones tercera, cuarta y quinta se dedicaron a estudiar, en la tercera, la revisión de las Ideas que se da en los años veinte en Friburgo; en la cuarta, el giro práctico de esos mismos ańos; y en la quinta, el problema del comienzo de la filosofía con el abandono del camino cartesiano y la apertura de nuevos caminos a la fenomenología que culmina en La crisis.
} 
rando la amplia y completa bibliografía de Steven Spileers (1999), tenemos acceso al recuento completo de todos los títulos de la obra publicada de Husserl. En total 54 títulos a lo largo de toda su vida. De estos, catorce en la época de Halle (la tesis doctoral fue presentada y publicada en Viena), con un total de mil cuatrocientas cuarenta y cuatro páginas. Otros catorce son de Gotinga, pero ahí están incluidos los dos primeros tomos de la segunda edición de las Investigaciones lógicas, publicados en 1913. El total cuatrocientas ochenta y ocho. De la época de Friburgo hay veinticinco entradas, en las que se incluye el tercer tomo de las Investigaciones lógicas, que recoge una muy reelaborada "VI Investigación", que sale en 1921, y las ediciones segunda y tercera de Ideas, de 1913, publicadas respectivamente en 1922 y en 1928, así como tres cartas. El número de páginas, quitando las entradas citadas últimamente, alcanza un total de seiscientas cincuenta y seis. Por tanto, el total de las páginas publicadas por Husserl, quitando las cartas y segundas o terceras ediciones, no llega a dos mil seiscientas. Pues bien, los manuscritos que Husserl dejó al morir, si ańadimos la correspondencia, eran cerca de cincuenta mil páginas. Los textos de investigación están escritos en la taquigrafía de Gabelsberger, un tipo de estenografía propia del alemán que se basa en la letra cursiva gótica alemana, por lo que resulta muy compleja para el que no tiene fluidez en el gótico alemán. Eso dificulta la lectura de los manuscritos de Husserl si no han sido previamente transcritos. De todos modos la proporción entre lo que Husserl publicó en vida y lo que escribió es del orden del cinco por ciento; o, dicho de otro modo, de cada diez páginas que escribió sólo publicó media página.

Eso no quiere decir que las publicadas no fueran importantes. De hecho, los pocos libros que publicó fueron decisivos: primero Investigaciones lógicas, al final de la etapa de Halle; luego, Ideas, poco antes de concluir la etapa de Gotinga. Y, por fin, La crisis, un año antes de la enfermedad que lo llevó a la muerte, por tanto 
como cierre de la etapa de Friburgo. Ahora bien, tanto Ideas, de 1913, como este último, La crisis, son entendidos como rupturas con las etapas anteriores, que tendrían consecuencias de cara a la comprensión de Husserl. En todo caso, es obvio que ha de resultar difícil entender la obra husserliana a partir de lo publicado cuando esto es sólo un cinco por ciento de lo escrito.

Lógica formal y trascendental, de 1929, es el texto que Husserl escribió en principio para prólogo de una recopilación de textos sobre la experiencia directa y el juicio que estaba haciendo Landgrebe a partir de manuscritos del maestro. Landgrebe, por entonces, al presentar su doctorado en 1928, dejó de ser ayudante de Husserl para ir a la Universidad alemana de Praga. El prólogo que Husserl escribió se convirtió en el libro sobre la lógica formal y trascendental. En cuanto al texto preparado por Landgrebe, no dio tiempo a sacarlo en vida de Husserl y por fin se publicó poco después de morir éste. Pero, como el propio recopilador y en gran medida redactor Landgrebe cuenta en el prólogo a la edición, al año siguiente, en la primavera de 1939, se produjo la anexión de Checoslovaquia al Tercer Reich, con lo que la edición quedó en Praga siendo destruida, excepto doscientos ejemplares que ya habían salido para Inglaterra y que fueron distribuidos sobre todo en los Estados Unidos de América. Con ello, el texto permaneció totalmente desconocido en el continente europeo. Así, la publicación de Experiencia y juicio apenas tuvo influencia en la configuración pública del pensamiento de Husserl.

Por otra parte, desde que Heidegger se fue a Marburgo su actuación contra Husserl en la cátedra fue muy continua y contundente. Desde la primera lección en el primer semestre que estuvo en Marburgo, en el invierno de 1923-1924, se dedicó con minuciosidad a criticar la fenomenología del que se pensaba que era su maestro. Los puntos fundamentales de esta crítica se pueden leer actualmente en las obras publicadas de esa época en la Gesamtausgabe. La primera 
lección de Heidegger en Marburgo, que se titula Einführung in die phänomenologische Forschung (Wintersemester 1923/24), ${ }^{5}$ está casi de modo pleno dedicada a criticar la forma en que Husserl presentó la fenomenología en las Ideas de 1913. El objetivo fundamental es mostrar que la fenomenología husserliana no va a las cosas mismas, sino a unas cosas que se constituyen en la mirada del científico, en una mirada, por tanto, contaminada por la preocupación por la seguridad del conocimiento: "Preocupación por el conocimiento conocido". Y es desde esa preocupación que Husserl, según Heidegger, da prioridad a la conciencia, destacando "el título conciencia sobre cualquiera de las otras vivencias". Desde esa perspectiva de que la conciencia es, como si dijéramos, una vivencia más, puede manifestar que decir que el amor es una conciencia-de es una monstruosidad, "eine Ungehenerlichkeit". La noción de conciencia que Heidegger descubre en Husserl, proveniente de la preocupación por el conocimiento conocido, es decir, por asegurar el conocer, lo encajona en la tradición cartesiana. Y aunque Heidegger subraya las diferencias entre Husserl y Descartes, lo que cuenta es que la conciencia husserliana es la misma que la cartesiana, un cogito me cogitare, lo que significa que la conciencia es siempre reflexión. Partiendo de ahí, para Heidegger, "la totalidad de las categorías fundamentales con las que está caracterizada la conciencia" quedan contaminadas, por ejemplo, la intencionalidad, la evidencia y la propia reducción eidética. ${ }^{6} \mathrm{La}$ primacía que da Husserl a la conciencia vendría de que detrás de toda otra vivencia está la vivencia del conocimiento, de manera que éste tiene una primacía, porque la intencionalidad siempre es concebida de modo teórico, porque "para todo conjunto de actos entramados el mentar teórico constituye el fundamento" (pág. 272). La razón de

\footnotetext{
${ }^{5}$ Gesamtausgabe, tomo XVII. Editada por Friedrich-Wilhelm von Herrmann, 1. Auflage 1994. 2. unveränderte Auflage 2006.

${ }^{6}$ Para una ampliación véase del autor el texto "La crítica heideggeriana a la fenomenología de Husserl”, en Ranch Sales y Pérez Herranz (editores), 1998, pp. 89-96.
} 
esta imputación está en que para Heidegger la conciencia husserliana está tallada por la conciencia cartesiana de carácter reflejo: el cogito me cogitare. Por tanto, todo se ve desde la óptica de la reflexión teórica. Y eso sería una perversión de la intencionalidad porque la intencionalidad está, entonces, dirigida por una intencionalidad teórica, olvidando en consecuencia otros tipos de intencionalidad. En las lecciones del semestre del verano de 1925, Prolegomena zur Geschichte des Zeitbegriffs ${ }^{7}$, ampliará esta visión de la fenomenología, de que la propuesta husserliana depende del horizonte cartesiano. Como el punto de vista cartesiano, que toma la conciencia como una vivencia reflexiva, no es originario, la fenomenología husserliana no va a lo originario. $^{8}$

Con estas lecciones de Heidegger se iría creando una visión convencional de Husserl, a la que también contribuirá éste mismo porque en absoluto tomará conciencia de las distorsiones de su pensamiento que podía haber en esa forma convencional en que estaba siendo entendido, y tardará mucho en reaccionar. Pero para entender bien el contraste con lo que llamamos el nuevo Husserl, deberemos tener claras algunas características de ese Husserl convencional, que en gran medida viene mediatizado por las ideas heideggerianas anteriores. Pero antes quiero aclarar la noción del "nuevo" Husserl.

\section{La noción del "nuevo" Husserl}

Antes de hablar del Husserl convencional, quiero aclarar la palabra "nuevo" aplicada a Husserl. Quiero aclarar, primero, que mi estudio de Husserl, al estar basado en una lectura de manuscritos, siempre se movió en una contraposición con ese Husserl conven-

\footnotetext{
7 Prolegomena zur Geschichte des Zeitbegriffs (Sommersemester 1925), editado por Petra Jaeger, $1^{a}$ edición, 1979; 2a edición revisada, 1988; 3a edición revisada, 1994.

${ }^{8}$ En la tercera sesión se aclaró el concepto de reflexión.
} 
cional que se basaba en los libros publicados en vida de Husserl, mediados o entendidos desde la perspectiva que se estaba instalando, procedente de las lecciones de Heidegger, que partía principalmente de las Ideas o, en general, de las obras publicadas en vida por Husserl. Toda mi vida profesional transcurrió en esa dualidad, la del Husserl que yo leía en los manuscritos y el Husserl que he llamado convencional. Naturalmente, y por suerte, yo no estaba solo en esa lectura, éramos muchos, tal como luego veremos. En realidad globalmente mi lectura se basaba en todos los que luego citaré.

Pero poco a poco se fueron publicando las obras de Husserl, de manera que muchos de aquellos manuscritos irían viendo la luz a lo largo de los años, por eso textos desconocidos en los años treinta o cuarenta, incluso en los cincuenta, se empezaban a conocer en los años sesenta. Así fue apareciendo aquel Husserl de los manuscritos, que resultaba muy diferente del Husserl convencional, pero con dos excepciones: el Husserl de los manuscritos coincidía en gran medida con el Husserl de La crisis y de La lógica formal y trascendental.

Precisamente, la publicación de la primera parte de La crisis, que abarca los $\$ \$ 1-27$-y las páginas 1-104 de la edición de Husserliana-, hizo difundirse rápidamente la idea de que en esa obra Husserl había dado un giro radical para responder a lo que había pasado en Alemania con la instauración del poder nacionalsocialista, que le llevó a Husserl a verse como un marginado en su propia tierra, y a ver su filosofía tachada como un producto de un no ario, ya que Husserl, como su mujer, aunque bautizados en la fe protestante, eran de origen judío. Esta es la imagen que perduró hasta muy avanzado el siglo XX, en todo caso mucho más allá de los años setenta. Cuando empecé a estudiar fenomenología, la imagen convencional era que había dos Husserl profundamente incompatibles, el de las Ideas y el de La crisis. "A mí me gusta el Husserl de La crisis", me decía un conocido profesor español. Husserl habría dado un giro hacia la historia y la política porque el nazismo fue 
tan violento y duro que nadie podía mantenerse indiferente frente a él, y menos un filósofo de origen judío.

Pero he dicho que poco a poco se fueron publicando los manuscritos de Husserl, y por fin aquella separación entre el Husserl convencional y el de los manuscritos -el Husserl convencional, aunque fuera dividido en un primer Husserl y un segundo Husserl opuesto-, ha ido siendo abandonada por los estudiosos porque bastaba con acudir a los textos publicados en los últimos veinte años para ver la inconsistencia de esa interpretación. Sin embargo, hay que decir que sigue plenamente instalada en la opinión pública filosófica $y$, muchas veces, en entornos científicos cuando tienen que citar algo concerniente a las posiciones del pensamiento. No es raro encontrarse con descalificaciones de la fenomenología por ser subjetivista o solipsista.

Y es ahí donde surgió la idea de un "nuevo" Husserl, cuando en 2003 el profesor norteamericano Donn Welton publicó un libro con ese título: The new Husserl. A Critical Reader. El libro recoge doce contribuciones en las que se analizan temas que habían sido generalmente mal interpretados en el Husserl convencional. Resalto este origen reciente para indicar que el llamado "nuevo" Husserl es el Husserl que los que leíamos sus manuscritos oponíamos al convencional y que, por fin, con un buen número de esos inéditos publicados en la colección Husserliana, era hora ya de oficializar. Ese Husserl que muchos estudiamos al adentrarnos en la fenomenología leyendo esos manuscritos es el "nuevo Husserl".

Por tanto, no se trata de un Husserl que recién se descubra sino uno que desde el principio estuvo ahí, y que por fin ya ha pasado a la opinión pública como el "nuevo" Husserl gracias a la feliz edición del libro de Welton y al acierto del título. Pero es importante no perder la perspectiva de que no es uno recién descubierto, sino el Husserl que todos cuantos lo estudiamos desde sus obras póstumas y sus manuscritos teníamos en mente. 


\section{Rasgos del Husserl convencional}

Vamos a centrarnos ahora en este Husserl convencional, porque es la mejor forma de comprender, frente a él, lo que representa el "nuevo". El primer rasgo del Husserl convencional viene a resultas del comienzo mismo de la fenomenología: la refutación del psicologismo; refutación que, llevada a cabo en los Prolegómenos para una lógica pura, de 1900, el primer tomo de las Investigaciones lógicas, quedará ampliada en ese mismo texto al antropologismo, del que el psicologismo no es sino un sucedáneo. El psicologismo se llama así porque estamos hablando de la relación de la Psicología con la Lógica y las Matemáticas. Pero, en realidad, estamos hablando de la mente humana, es decir, de aquello que hace al humano tal, por tanto, de la historia de este humano. Y ahí entra el antropologismo. La fenomenología, al rechazar expresamente el psicologismo, está rechazando el antropologismo y, en general, la Antropología como punto de partida de cualquier explicación en el sistema del saber.

Eso significa que la fenomenología no trata del ser humano - del que se ocupa la Antropología- sino de un sujeto que no es el ser humano. Esta cuestión, que en las Investigaciones lógicas parece no tener excesiva importancia, se irá complicando hasta el final de la vida del Husserl. Porque no es un tema sin importancia, pues de hecho tiene consecuencias positivas y negativas de largo alcance. Positivas porque, al trascender del ser humano, la fenomenología se sitúa en un nivel muy por encima de todo subjetivismo. Porque el antropologismo es, en definitiva, asentarse en un subjetivismo de la especie. Según él, las verdades serían siempre meramente nuestras verdades, pero no necesariamente lo son para Dios o para otros seres racionales. Eso significa que, por ejemplo, una explosión nuclear podría serlo sólo para nosotros, pero no para otros seres racionales. Pues bien, la fenomenología, al rechazar el 
psicologismo, está rechazando todo antropologismo y, consecuentemente, que la Filosofía o, en este caso, la fenomenología, que se postula como Filosofía primera, sea una Antropología. Este tema recorrerá en gran medida la obra de Husserl.

Como consecuencias negativas podemos citar: la ventaja que parece tener la fenomenología al deslindar los campos de trabajo, acarrea a la vez un fuerte inconveniente, porque no tratar del ser humano le obligará a decir de quién trata. En las Investigaciones lógicas quedará pendiente la respuesta a esta pregunta. Pues si no se trata del ser humano, es obvio que se trata de alguien. Por fin dirá Husserl que trata del sujeto trascendental.

Pero en seguida surgirá la duda de quién es el sujeto trascendental, dónde está y qué hace si es que hace algo. En fin, tanto la pregunta como la respuesta llevaron a la fenomenología por un derrotero sumamente incómodo, sobre todo cuando ya a principios de los años veinte, Heidegger, colaborador de Husserl, está analizando conductas muy precisas y concretas del ser humano en su más estricta facticidad. En Ser y tiempo incluso propondrá para comienzo de sus análisis el ser humano en su cotidianidad, es decir, en las actuaciones más inmediatas en las que nos encontramos. Husserl, al contrario, hablaba de cosas abstrusas, totalmente ajenas a nuestra vida ordinaria.

Segundo. El carácter abstracto del sujeto estudiado por la fenomenología se ratifica también desde otra perspectiva, a saber, desde la lucha contra el historicismo que llevó a cabo Husserl ya en el artículo de Logos, "La filosofía como ciencia rigurosa", en el que refuta tanto el naturalismo como el historicismo, porque, según éste, los productos de las ciencias formales y, en definitiva, los de las ciencias en general, son históricos, no sólo en el sentido de que son irremediablemente producidos en la Historia, sino también en el sentido de que el tiempo en el que esos productos valen es limitado, bien el tiempo de la época del que lo formula 
o durante unas cuantas generaciones, bien el tiempo que dura la historia de un pueblo o una coyuntura determinada, etc., como puede ser histórico un sistema de gobierno, pongamos por caso, la monarquía. Husserl no tiene más que aplicar la refutación del psicologismo. Pero con este ataque al historicismo Husserl parecía alejarse de la Historia, lo que se ratifica con el hecho de que en su obra publicada no había ninguna referencia histórica. Parecía, por tanto, que el sujeto husserliano planeaba por encima de la historia, y que, por tanto, era ahistórico. La característica de que la fenomenología no se preocupa por la Historia ha sido uno de los tópicos más pertinaces de la historia de la fenomenología, incluso mucho después de haberse publicado textos que demostraban exactamente lo contrario (véase Díaz Álvarez, 2003).

En esta consideración, Ortega es un ejemplo de primer rango. Es razonable pensar que Ortega tuvo conocimiento relativamente pronto de la publicación por parte de Husserl de la primera sección de La crisis, en 1936, porque en París coincide con Zubiri, en quien ese texto había encontrado una fuerte resonancia. Teniendo en cuenta que ambos coincidieron en París, es fácil pensar que comentaran las novedades de ese texto. Al finalizar la Guerra Civil Ortega viajará a Argentina, donde publicará un texto con el título "Apuntes sobre el pensamiento: su teurgia y su demiurgia” (OC, VI, 3-29). El objetivo del texto es enraizar el pensamiento en la vida. El ensayo, muy interesante por sí mismo, tiene también gran interés historiográfico porque es el primer texto publicado de Ortega en el que aparece una crítica a Husserl -por más que la estuviera ejerciendo en su docencia desde 1929-. En ese texto Ortega quiere exponer la diferencia de su enfoque con el de Husserl, porque éste se muestra ajeno a la vida porque es un pensador que, centrado en las esencias, no puede acercarse a la vida ni a la historia. Precisamente, en la Lógica formal $y$ trascendental se ve un rasgo que desmiente esta afirmación de Ortega porque ahí Husserl empieza, lo reconoce Ortega, aludiendo a la 
situación del momento en el que se detecta una pérdida de la fe en la razón y, como Husserl ya lo ha dicho, esa es también una pérdida de la fe en la razón práctica. A Ortega no le preocupa este acercamiento de Husserl a la historia porque sólo ocurre en esa ocasión. Y la razón es que esa pérdida es tan fuerte que ha "penetrado hasta su [de Husserl] retiro" (OC, VI, 7), traspasando así los muros de la ahistórica torre de marfil en que estaba encerrada la fenomenología. Una vez terminado el texto, Ortega añade una nota en la que dice que le acaba de llegar la última publicación de Husserl, a saber, la parte publicada de La crisis, que toda ella es un relato histórico, porque trata de la crisis de las ciencias que está ocurriendo en ese momento. Ahí ya no es una anécdota, como se podía decir de La lógica, porque aquí el tema es un tema histórico. Si eso fuera así, la fenomenología no estaría incapacitada para acercarse a la historia como acababa de decir Ortega. Pero ahora tiene una buena salida. Después de decir que esa obra de la filosofía husserliana "consiste nada menos que en recurrir a la... "razón histórica»" (OC, VI, 29), que es la filosofía propia de Ortega, con lo que sus filosofías estarían muy cercanas, y dado que con ello lo anterior del artículo quedaría invalidado, Ortega insiste en que no tiene nada que corregir porque ese texto publicado por Husserl en realidad no es de éste sino de su discípulo Fink, lo que es posible advertir por el lenguaje y los planteamientos.

Hasta ese punto estaba consolidada la opinión sobre la imposibilidad de la fenomenología de Husserl de acceder a la historia. En mi opinión, la forma en que Ortega trata el texto de La crisis al final es un modo retórico, porque es muy difícil que él, que estaba perfectamente informado de las grandes novedades filosóficas, no tuviera conocimiento de ese texto hasta haber escrito el artículo "Sobre el pensamiento...", tanto más cuanto había coincidido en París con Zubiri. Lo más probable es que, al tener la salida de la atribución del texto a Fink, ya pudiera poner su comentario fuera del texto principal. De todas maneras creo que hay que sacar im- 
portantes consecuencias del hecho de que la afirmación de Ortega es totalmente errónea, porque el texto es íntegramente de Husserl. ${ }^{9}$

Aún podemos ver una tercera consecuencia. Tal vez sea Husserl el primer filósofo en la historia de la Filosofía que ha elevado a rango prioritario el tema del otro. También lo hizo Fichte, pero Husserl lo hace con decisión y gracias a él entra en la Filosofía el tema de la intersubjetividad. No es un tema que aparezca desde el principio, pero lo hace temprano y también temprano lo resuelve o propone una solución. Sin embargo, el hecho de que el fenomenólogo sea, en este caso, yo o, en su caso, cada uno de ustedes, de manera individual, lleva a que las vivencias, las certezas y la reflexión sean siempre individuales, y que, elevadas a método, sea difícil proponer ahí, o ver ahí cómo se pone o introduce al otro. Porque ahora resulta que, en el sentido de la verdad o del bien, está que son verdad y bien para cualquiera, para todos. Si una verdad es para mí y la tomó como no siendo verdad para otro, la tomo como una no verdad, como algo que tomo como si estuviera en el limbo, porque puede no ser verdad. Por tanto, una verdad auténtica lo es para cualquiera. Eso significa que previamente tengo que tener el horizonte de los demás.

Y ¿cómo se me da ese horizonte si la reflexión es sólo mía? Por tanto, la intersubjetividad, el horizonte de los otros, está en la preocupación de la fenomenología desde el principio. Pero Husserl no publicó nada al respecto, ${ }^{10}$ y cuando publicó algo, ya muy tar-

\footnotetext{
${ }^{9}$ De estas consecuencias hablo en el capítulo $\mathrm{V}$ de mi libro La fenomenología de Ortega $y$ Gasset (2012). En efecto, teniendo en cuenta que no es cierto que el texto provenga de Fink, la filosofía de Ortega es semejante a la del último Husserl. Como éste coincide con el "nuevo" Husserl, la filosofía de Ortega coincide en gran medida con la del "nuevo" Husserl.

${ }^{10}$ La primera vez que plantea, y en parte resuelve, el problema es en las lecciones de 1910-1911. Véase la obra Problemas fundamentales de fenomenología, traducción de César Moreno y Javier San Martín. Introducción de J. San Martín. El texto alemán se publicó por primera vez en 1973 en Husserliana XIII.
} 
de, en las Meditaciones cartesianas, en francés, adoptaba un modo de hacerlo desde el planteamiento del modo cartesiano de acceso a la fenomenología, que parecía, más que posibilitar, incapacitar el pensamiento de la intersubjetividad. Por tanto, se acusó a la fenomenología de caer en el solipsismo. El fenomenólogo era un filósofo que se centraba en un sujeto solipsista, ${ }^{11}$ por lo que, si la fenomenología era impotente ante la Historia, mucho más lo era ante la sociedad. La fenomenología se quedaba vinculada al reino de la soledad, en un solipsismo metafísico. No importaba que Husserl remarcara que en la vida ordinaria somos humanos entre otros humanos. En realidad la fenomenología era, y es, una filosofía que sólo funcionaba de modo solipsista.

En realidad estas características se debían al cartesianismo de Husserl que, adoptado en las Ideas de 1913, queda ratificado en la Sorbona de París, en 1929, al pronunciar sus Pariser Vorträge (conferencias de París), donde Descartes es el pensador en que se fija Husserl, si bien a poco de empezar diga expresamente que apenas puede acompañar a Descartes porque éste inmediatamente había malinterpretado su descubrimiento. De todos modos el Husserl convencional quedó vinculado al cartesianismo de la Modernidad, siendo propio de él un cartesianismo incluso más acentuado porque es más consciente y está mejor articulado.

Todo este Husserl convencional utilizado en casi todas las refutaciones que se suelen hacer de Husserl, ha quedado como el Husserl que hay que contraponer, primero, al "nuevo" Husserl. Y segundo, es el Husserl que antes de surgir la denominación del "nuevo" Husserl, era el "primer" Husserl frente al de La crisis, que sería el segundo. Y por supuesto era el Husserl convencional contra el que cuantos estudiábamos los manuscritos de Husserl teníamos

\footnotetext{
${ }^{11}$ Así lo interpreta Gaos en el prólogo que escribió para la traducción de las Meditaciones cartesianas. (Véase la edición del Fondo de Cultura Económica, 1942: 34). También en Obras completas VII.
} 
que defendernos, porque nos parecía que ese Husserl convencional no era el que aparecía en los manuscritos. Por lo que los textos publicados habían de ser interpretados de otra manera.

\section{La historia del "nuevo" Husserl}

Una vez explicadas las dualidades que se han dado en la comprensión del Husserl, vamos a ver ahora la historia del nuevo Husserl, esa historia de la que somos protagonistas todos cuantos nos hemos acercado a Husserl desde sus manuscritos. Porque en su momento dije que no estábamos solos, que había muchos que nos habían precedido. En primer lugar tengo que citar a los discípulos de Husserl de la primera hora. Pero aquí hay que hacer una diferencia entre los discípulos de la Universidad de Gotinga y los de Friburgo. Ya he dicho que muchos alumnos del Husserl de Gotinga lo abandonaron después de aparecer, en 1913, las Ideas, porque creían que con ese libro Husserl abandonaba la postura realista de la etapa anterior. Esa evaluación hecha de las Ideas, como una filosofía trascendental al estilo kantiano, que se aleja de las cosas, sería definitiva. Por tanto, en cierta manera el paradigma (llamémosle así) del Husserl convencional, el primer Husserl ${ }^{12}$ frente al último Husserl, se fragua a partir de la publicación de las Ideas de 1913; y, luego, como ya lo hemos indicado, por las interpretaciones de Heidegger, por ejemplo, respecto al ser de la conciencia que, según Heidegger, es tomada o analizada como un objeto en virtud de la teoría de la reflexión, con lo que Husserl terminaría él mismo cosificando la conciencia; y, en segundo lugar, por trabajar con una mirada científica que contamina las cosas, de manera que en los análisis toma como directa una percepción que no se corresponde con la

${ }^{12}$ El concepto de "primer Husserl” no tomaría en consideración al Husserl realista de las Investigaciones lógicas, porque la fenomenología que ha pasado a la opinión es la del Husserl de las Ideas. 
percepción tal cual es. Heidegger acusará a Husserl de cometer una falacia metodológica, meter en las cosas lo que es resultado del método: tomar como directa una percepción que sólo será la percepción procedente del interés del científico, a la que por tanto se ha eliminado todo predicado humano.

Pero esto de Heidegger ocurre en los años de Marburgo, que coincide con la estancia de Husserl en Friburgo, adonde Husserl fue en 1916, y que es una etapa en la que revisa muy a fondo los resultados anteriores. Varios discípulos conocen estas revisiones. Primero, Edith Stein; luego, Ludwig Landgrebe; en tercer lugar, Eugen Fink. Junto con ellos también toma nota de los cambios Aron Gurwitsch, e incluso Roman Ingarden, que, en una muy importante anotación al texto de la traducción francesa de las Meditaciones cartesianas, que se escriben, como ya he dicho, en la época de Friburgo, le comentará a Husserl la novedad, en relación con las Ideas, del $\$ 32$, que se titula "El yo como sustrato de habitualidades". Román Ingarden, discípulo de la época de Gotinga, que siguió en relación personal con Husserl, le comenta este párrafo como "una novedad en relación con las Ideas" (Hua I, 215). Y para que no quede duda, después de unas interesantes reflexiones, le pide a Husserl que ratifique algo, por otro lado obvio si se toma en consideración la estructura de las Meditaciones cartesianas, porque el párrafo que aporta esa novedad es de la "IV Meditación", en la que se analiza el yo trascendental, por tanto, la habitualidades constituyen el yo trascendental. Pues bien, aun sabiendo esto, Roman Ingarden le comenta a Husserl que hay que fundamentar mejor "que las "propiedades habituales» pertenecen al yo puro, transcendental, y no al "psicológico», «humano»" (Hua I, 218). Si las habitualidades pertenecen al yo, evidentemente éste es un yo histórico cuya historia es un ir logrando configurarse como el yo de esas habitualidades. Éstas no son sino los hábitos con que conocemos, evaluamos y nos comportamos en el mundo, y que son resultado siempre de la reite- 
ración de esos mismos actos. Por otro lado, esa posición de Husserl, subrayada claramente por Roman Ingarden, indica un completo distanciamiento de la noción de yo trascendental de Kant, pues el yo trascendental husserliano somos cada uno de nosotros de modo individual, que no único, porque todo el que puede decir "yo" es yo trascendental. Veremos en este punto la deriva de Gaos.

Junto con los citados, también está otro pensador importante, el profesor norteamericano Dorion Cairns, que sostuvo importantísimas conversaciones con Husserl y Eugen Fink. La influencia de D. Cairns en Estados Unidos fue inmensa a la hora de configurar una imagen de Husserl alejada de la convencional. Por supuesto, junto con ellos hay que citar a Eugen Fink, que sustituyó a Landgrebe como ayudante de Husserl. Fue Fink quien escribió un muy importante artículo para distanciar la fenomenología del neokantismo en que generalmente queda incluida. ${ }^{13}$ En ese artículo aparecen ya puntos fundamentales de lo que por entonces escribió en la VI Meditación" cartesiana y que, aunque se publicó muy tarde (en 1988), de hecho pudo influir en la filosofía francesa a través de una copia que se movió por los círculos fenomenológicos de Francia, incluido Merleau-Ponty, cuyas obras deben entenderse desde las aportaciones de Aron Gurwitsch y Eugen Fink (véase Pintos Peñaranda, 2005, especialmente 117 y ss.).

Todos estos autores vivieron la reflexión de Husserl desde dentro, conociendo sus manuscritos y los problemas que Husserl tenía para transmitir la novedad de su filosofía muchas veces con palabras de la tradición. Por supuesto, su visión estaba profundamente alejada de la del Husserl convencional. La visión que ellos represen-

\footnotetext{
13 "Die phänomenologische Philosophie Edmund Husserls in der gegenwärtigen Kritik", en Kant-Studien 38 (1933: 319-383). También en Fink (1966). Hay una traducción al español de Raúl Eusebio Velozo Farías, "La filosofía fenomenológica de Edmund Husserl ante la crítica contemporánea”, en Acta fenomenológica latinoamericana, volumen 1, Lima, Pontificia Universidad Católica del Perú, 2003, pp. 361-428.
} 
taban procedía de aclaraciones de Husserl que permitían entender expresiones del Husserl de la época de Gotinga.

Así, a través de esos autores llegó ese otro Husserl, primero en los textos de Merleau-Ponty, quien aún podía tener dudas sobre el sentido último de la fenomenología o de expresiones de Husserl. Luego, ya en 1950, Gerd Brand (1921-1979) termina su tesis doctoral realizada bajo la dirección de Leo van Breda, con el título "Der Rückgang auf das welterfahende Leben und die Zeitlichkeit als seine Urform" [La vuelta a la vida que tiene experiencia del mundo y la temporalidad como su forma], y que pronto, ya en 1955, se publicó con el título de Welt, Ich und Zeit. Nach unveröffentlichten Manuskripten. En el texto se manejan manuscritos clave tanto del grupo C, sobre las últimas investigaciones sobre el tiempo, como del grupo $\mathrm{B}$, en concreto maneja el manuscrito B I 5 IX, en el que dice Husserl que en la medida en que vayamos progresando en la "explicitación de la concreción de este yo... el desasimiento [die Enthaltung] de la posición del mundo queda sistemáticamente superado" (1955: 34), es decir, la epojé aparece como algo provisional que puede ser superado.

Después vendrán los italianos, liderados sobre todo por Enzo Paci, discípulo a su vez de Antonio Banfi, quien introdujo a Husserl en Italia, iniciando una tradición fenomenológica vinculada a Husserl y a la izquierda italiana. Banfi llegó a conocer a Husserl, en 1930, durante la estancia de éste en Chiavari. A Banfi sucedió en la cátedra de Milán Enzo Paci, que, además de crear y dirigir la revista Aut aut, escribió importantes contribuciones a la fenomenología desde el Husserl de La crisis. Pero lo que más nos interesa aquí es que procuró que sus discípulos fueran a Alemania o Bélgica a hacer sus respectivas tesis doctorales a partir de manuscritos de Husserl. Así lo hizo Giovanni Piana (1940), que publicó su tesis Esistenza e storia negli inediti di Husserl (Lampugnani Nigri, Milano, 1965). Resultado de este conocimiento de los manuscritos de Husserl fue la rápida traducción al italiano del libro antes citado de 
Gerd Brand, que se publicó en italiano ya en 1960. El jesuita Ponsetto, por su parte, estudió en Colonia con Landgrebe, fundando la serie periódica Fenomenologia e Società, en la que se insiste en la vertiente práctica de la fenomenología.

En Alemania el fenomenólogo más importante que se centró desde el principio en el nuevo Husserl y en sus manuscritos fue Klaus Held (1936), que hizo la tesis doctoral sobre la "presencia viviente", la "Lebendige Gegenwart", basándose sobre todo en los manuscritos C sobre el tiempo. Luego, la habilitación la escribió sobre Heráclito, con lo que tuvo una inmejorable base para entender la fenomenología desde una perspectiva histórica. Por su parte, Bernhard Wandelfels (1934) entró en la fenomenología del nuevo Husserl más por la fenomenología francesa, de la que se convirtió en principal defensor en Alemania, que por el estudio directo de los manuscritos. A partir de 1962, su estancia en París debió de ser decisiva para configurar el contenido de su fenomenología, que también responde a la idea del nuevo Husserl.

\section{Las anotaciones de Gaos en el Congreso sobre el mundo de la vida}

Para terminar quiero confrontar al nuevo Husserl con el convencional a partir de las intervenciones de Gaos en los años sesenta, que prolongan las notas que escribió en 1959 para la sesión pública en homenaje a Edmund Husserl con motivo del centenario de su nacimiento. El texto elegido para la conmemoración fue "La filosofía como ciencia rigurosa", y en él participaron los miembros del Seminario de Filosofía Moderna de la Facultad de Filosofía de la Universidad Nacional Autónoma de México (véase Zirión, 2003: 197). Para la publicación de esas contribuciones, el que entonces era director del seminario, José Gaos, redactó unas notas que debían haber salido en 1961, pero que aparecieron realmente en 1963 
(véase Salmerón, 2006: 248, nota 52). Las notas son una especie de ajuste de cuentas con Husserl. En este contexto es muy interesante considerar lo que ocurrió en México en esos años, porque fue el momento en que la fenomenología prácticamente desapareció de México al "rebelarse" los discípulos de Gaos frente a su maestro. ${ }^{14}$ Sus alumnos Alejandro Rossi y Luis Villoro habían defendido la contemporaneidad de Husserl. Gaos, sin contestar a sus discípulos, arremete de modo contundente contra Husserl, cuya filosofía científica es "un monstruo de contradicciones" (Gaos, Notas, OC, $\mathrm{X}, 66$, nota 4).

En 1963 tuvo lugar en México, en el seno del XIII Congreso Internacional de Filosofía, el "Simposio sobre la noción husserliana de la Lebenswelt" ${ }_{15}^{15}$ que por la asistencia de fenomenólogos fue de máximo relieve en el mundo de la fenomenología. A él asistieron miembros muy significados de ese movimiento, como Ludwig Landgrebe, de Alemania, ayudante de Husserl en los años veinte y que, luego, junto con Eugen Fink enseñarían a transcribir los manuscritos de Husserl; y Enzo Paci, de reconocido prestigio no sólo en Italia sino también en otros países. De Estados Unidos vino John Wild; de México se alineaba con estos fenomenólogos el discípulo de Gaos,

${ }^{14}$ De "la rebelión de los alumnos" habla Salmerón (2006: 243; véase también Isegoría, 1, 1991: 129). Salmerón se refiere a que Gaos, en la conferencia sobre "Antropología filosófica" (1965), habla de los discípulos rebeldes en otro contexto -que es el de los discípulos rebeldes de Husserl (Gaos, 1967: 20)-, pero Salmerón se refiere a que Gaos "hace público lo que considera el contenido esencial de las discusiones privadas con sus propios discípulos" (Salmerón, 2006: 243), aludiendo ahí a la rebelión de sus alumnos. El hecho es que al menos los tres alumnos más importantes de Gaos que empezaron vinculados a la Fenomenología (Rossi, Villoro y el mismo Salmerón), poco después, en 1967, crearon la revista Crítica que terminaría siendo una publicación de tendencia analítica (Salmerón, 2006: 249 y ss., específicamente la 351). Véase también Zirión (2003: 323).

${ }^{15}$ Antonio Zirión comenta que "Quizá éste fue el último evento de significación fenomenológica en México antes del oscurecimiento de aproximadamente 16 años que siguió al último curso de fenomenología de Husserl que impartió Villoro en la Facultad de Filosofía y Letras de las unAm, y que podemos situar entre 1964 y 1965.” (2003: 206, nota 41). 
Luis Villoro. El objetivo del congreso debía ser poner a punto la fenomenología después de la publicación del tomo VI de Husserliana, La crisis de las ciencias europeas, que tuvo lugar en 1954.

Para entender lo que este congreso representaba, hay que tomar en consideración que de La crisis se habían publicado en 1936 las dos primeras partes, que abarcan los $\$ \$ 1-27$, y las páginas 1-104, de la nueva edición. A partir del $\$ 28$ ya es todo inédito, y precisamente en ese parágrafo se empieza a hablar del problema del mundo de la vida como el presupuesto implícito de Kant, y al hablar de ese mundo, ya en ese parágrafo, se mencionan reiteradamente las cinestesias como elementos fundamentales en la aparición del mundo de la vida presupuesto en las ciencias. En ese parágrafo la palabra "cinestesias" aparece hasta siete veces. Lo que no puede pasar desapercibido a un lector normal.

Es cierto que desde el Husserl cartesiano esas cinestesias, como pertenecientes al cuerpo, han tenido que ser desconectadas para lograr la subjetividad trascendental o la conciencia pura. Pero los $\$ \$$ 41, 42 y 43 de La crisis están dedicados a la práctica de la epojé y la reducción trascendentales, con lo que tenemos que dar por hecho que los análisis posteriores ya son de la subjetividad trascendental. Pues bien, a partir del $₫ 47$ vuelven a aparecer las cinestesias, ahora como Grundphänomene, "fenómenos fundamentales" (Hua VI, 163), y ya no lo son de la subjetividad empírica de antes de la reducción, sino de la subjetividad trascendental, porque estamos después de la reducción. Por tanto, las cinestesias, con las que está íntimamente conectada la visión del cuerpo real, pertenecen a la subjetividad trascendental. Este es el problema que planteaban esas páginas en las que la palabra "cinestesias" aparece reiteradamente ya en el plano trascendental, con lo que aquellas visiones que se tenían de la subjetividad trascendental contaminada de kantismo -como es el caso de Gaos-, tienen que ceder a una nueva visión, en la que la subjetividad trascendental tiene un mundo vinculado 
a los sentidos corporales de nuestra realidad concreta y a las diversas sensaciones cinestésicas. Eso solo es pertinente si se habla de la subjetividad trascendental como de la subjetividad de cada uno de nosotros, porque sólo así es posible analizar las sensaciones cinestésicas que pertenecen a la vida concreta de cada uno. Los que vienen a México de fuera han asumido esas tesis de Husserl, o quizás nunca tuvieron otra porque conocían a Husserl desde dentro de su práctica filosófica. Gaos, en cambio, se aferraba a su noción del Husserl convencional. Las diversas anotaciones que hace muestran la violencia del choque entre estas dos concepciones. Nada más interesante para visualizarlas que leer con detenimiento tanto la comunicación de Gaos como, sobre todo, las anotaciones que hace a las comunicaciones de los demás, que ahora se nos han hecho accesibles en el tomo X de las Obras Completas de Gaos.

\section{Bibliografía}

Brandt, G., 1955, Welt, Ich und Zeit. Nach unveröffentlichten Manuskripten, La Haya, Martinus Nijhoff.

Bolado, Gerardo, 2005, "La renovación institucional de la filosofía en España después de Ortega", en Ortega en circunstancia. Una filosofia del siglo XX para el siglo XXI, (J. San Martín y J. Lasaga, editores), Madrid, Biblioteca Nueva/Fundación José Ortega y Gasset, pp. 15-41.

Fink, Eugen, 1933, "Die phänomenologische Philosophie Edmund Husserls in der gegenwärtigen Kritik", en Kant-Studien 38 [hay una traducción al español de Raúl Eusebio Velozo Farías, 2003, "La filosofía fenomenológica de Edmund Husserl ante la crítica contemporánea”, en Acta fenomenológica latinoamericana, vol. 1, Pontificia Universidad Católica del Perú, pp. 361-428]

Nijhoff.

, 1966, Studien zur Phänomenologie 1930-1939, La Haya, 
Gaos, José, 1999, Obras completas: De Husserl, Heidegger y Ortega, tomo X, (prólogo de Laura Mues de Schrenk; edición coordinada por Antonio Zirión Q.), México,unam.

, 1942, "Prólogo: historia y significado", Meditaciones cartesianas, México, FCE, pp. 27-39.

Heidegger, Martin, Einführung in die phänomenologische Forschung (Wintersemester 1923/24), Gesammtausgabe, tomo XVII, (editada por Friedrich-Wilhelm von Herrmann) [1 ${ }^{\text {a }}$ ed., 1994; 2a ed., 2006].

, 1979. Prolegomena zur Geschichte des Zeitbegriffs (Sommersemester 1925), $1^{\text {a }}$ ed., (editado por Petra Jaeger, Gesammtausgabe), tomo XX, [2 a ed. revisada, 1988; $3^{\mathrm{a}}$ ed. revisada, 1994].

Hopkins, Burt, 2001, "The Husserl-Heidegger Confrontation and the Essential Possibility of Phenomenology: Edmund Husserl, Psychological and Transcendental Phenomenology and the Confrontation with Heidegger", en Husserl Studies 17, pp. 125-148.

Husserl, Edmund, 1973, „Nr. 6. Aus den Vorlesungen, Grundprobleme der Phänomenologie". Winterse-mester 1910/11, en Hua XIII, pp. 111-194. [Trad. castellana, Problemas fundamentales de fenomenología, (trad. de C. Moreno y J. San Martín. Introducción de J. San Martín), España, Alianza Editorial, 1994.]

Ortega y Gasset, José, 1942, “Apuntes sobre el pensamiento: su teurgia y su demiurgia", en Obras completas, tomo VI, Madrid, Taurus.

Pintos Peñaranda, M. Luz, 2005, "Husserl und Merleau-Ponty. Schlüssel einer engen Beziehung", en Phänomenologie in Spanien, (edición de Javier San Martín), Würzburg, Koenigshausen \& Neumann, pp. 111-125.

Salmerón, Fernando, 2006, Gaos y la filosofía Iberoamericana, segunda parte, México, El Colegio Nacional.

San Martín, Javier, 2012, La fenomenología de Ortega y Gasset, Madrid, Biblioteca Nueva. 
, 2007, "La recepción de Ortega en España. Al rebufo de un error autobiográfico", en Ortega en pasado y en futuro: medio siglo después (edición de J. Lasaga, M. Márquez, J. M. Navarro y J. San Martín), Madrid, Biblioteca Nueva.

, 2005, Phänomenologie in Spanien, (J. San Martín, Hrsg.), Würzburg, Koeningshausen \& Neumann.

, 2005, “Ortega y Gasset, Cervantes y Don Quijote”, en Meditaciones sobre Ortega y Gasset, (edición de Fernando Llano y Alfonso Castro), Madrid, Editorial Tébar, pp. 193-232.

, 1999, Fenomenología y cultura en Ortega, Madrid, Tecnos.

, 1998, "La crítica heideggeriana a la fenomenología de Husserl", en Seminario de Filosofía. Centenario René Descartes (15961996), (edición de E. Ranch Sales y F. M. Pérez Herránz), Alicante, Universidad de Alicante, pp. 89-96.

, 1994. Ensayos sobre Ortega, Madrid, UNED.

Spileers, Steven, 1999, Edmund Husserl Bibliography, (compilado por Academic Publisher), Dordrecht / Boston / London.

Welton, Donn, 2003, The new Husserl. A Critical Reader, Bloomington, Indiana University Press.

Zirión, Antonio, 2003, Historia de la fenomenología en México, Morelia, Jitanjáfora.

Zubiri, Xavier, 1980, Cinco lecciones de filosofía, Madrid, Alianza Editorial. 